\title{
EMG-Based Analysis of the Upper Limb Motion
}

\author{
Iason Batzianoulis, \\ Sahar El-Khoury \\ Learning Algorithms and \\ Systems Laboratory \\ Ecole Polytechnique Fédérale \\ de Lausanne, Switzerland \\ iason.batzianoulis@epfl.ch \\ sahar.elkhoury@epfl.ch
}

\author{
Silvestro Micera \\ Translational Neural \\ Engineering Lab \\ Ecole Polytechnique Fédérale \\ de Lausanne, Switzerland \\ The BioRobotics Institute \\ Scuola Superiore Sant'Anna, \\ Pisa, Italy \\ silvestro.micera@epfl.ch
}

\author{
Aude Billard \\ Learning Algorithms and \\ Systems Laboratory \\ Ecole Polytechnique Fédérale \\ de Lausanne, Switzerland \\ aude.billard@epfl.ch
}

\begin{abstract}
In a human robot interaction scenario, predicting the human motion intention is essential for avoiding inconvenient delays and for a smooth reactivity of the robotic system. In particular, when dealing with hand prosthetic devices, an early estimation of the final hand gesture is crucial for a smooth control of the robotic hand. In this work we develop an electromyographic (EMG) based learning approach that decodes the grasping intention at an early stage of the reaching to grasping motion, i.e before the final grasp/hand preshape takes place. EMG electrodes are used for recording the arm muscles activities and a cyberglove is used to measure the finger joints during the reach and grasp motion. Results show that we can correctly classify with $90 \%$ accuracy for three typical grasps before the onset of the hand pre-shape. Such an early detection of the grasp intention allows to control a robotic hand simultaneously to the motion of subject's arm, hence generating no delay between the natural arm motion and the artificial hand motion.
\end{abstract}

\section{Categories and Subject Descriptors}

I.2.6 [Learning]: Knowledge Acquisition; I.5.4 [Pattern Recognition]: Applications- Signal processing; H.1.2 [User / Machine Systems]: Human information processing

\section{General Terms}

Human Factors, Experimentation

\section{Keywords}

Human grasp, Hand preshape, Electromyography, Echo State Networks

\section{INTRODUCTION}

Electromyographic (EMG) signals provide information related to the muscles activity and has been used for estimating the user's motion intention. In particular, several EMG

Permission to make digital or hard copies of part or all of this work for personal or classroom use is granted without fee provided that copies are not made or distributed for profit or commercial advantage and that copies bear this notice and the full citation on the first page. Copyrights for thirdparty components of this work must be honored. For all other uses, contact the Owner/Author.

Copyright is held by the owner/author(s)

HRI'15 Extended Abstracts, Mar 02-05 2015, Portland, OR, USA

ACM 978-1-4503-3318-4/15/03.

http://dx.doi.org/10.1145/2701973.2701997 .
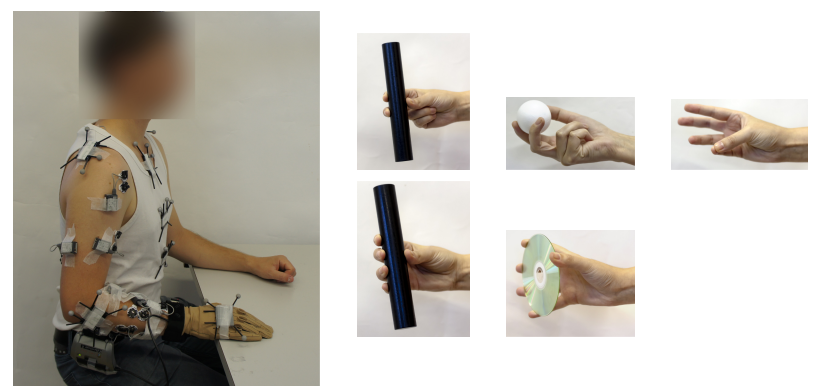

Figure 1: The experimental setup showing the electrodes for EMG recording, the vision tracking system for computing the arm joint angles and the Cyberglove for capturing the hand joint angles as well as the tested grasp types.

based systems were proposed for estimating upper limb motions. Some papers classify among different static hand gestures [6] or reaching and grasping motions in [3, 2]. Studies show that humans select few prehensile postures or preshapes, at an early stage of the reach and grasp motion, before the hand reaches the object [5]. No previous study correlates the classification performance of the reaching and grasping motion to the one of the hand preshape. This study contributes to the current state of the art of EMG analysis of the upper limb motion by proposing an EMG based learning approach that decodes the grasping intention of the user at an early stage of the reaching to grasping motion, enabling a smooth human robot interaction.

\section{EXPERIMENTAL PROTOCOL}

Five right handed healthy subjects, all volunteers (four males and one female), whose age is between $26-38$, participated in the experiment.

During the experiment, the subjects sat straight with their right hand flat on the table and pointing toward the object, see Figure (1). The subjects were then asked to reach for the object and grasp it with a predefined grasp type. The object was placed $30 \mathrm{~cm}$ away from the initial hand position. Data from 15 EMG electrodes (Superior Trapezius, Deltoid Anterior, Biceps Brachii long heads, Triceps Brachii long head, Pronator Teres, Flexor Digitorum Superfialis, Extensor Digitorum Communis, Flexor Carpi Ulnaris, Extensor Carpi Ulnaris, Flexor Carpi Radialis, Extensor Carpi Radialis longus, Flexor Pollici Brevis, Extensor Pollicis Bravis, Adductor Pollicis Transversus, Abductor Digiti Minimi) placed on the upper limb was recorded 
using the Noraxon DTS desktop system, with a sampling rate at $1500 \mathrm{~Hz}$. The joint angles of the fingers were measured using the Cyberglove, with a sampling rate of $280 \mathrm{~Hz}$, see Figure (1) left. Five grasp types (precision disk, tripod, thumb-2fingers, thumb-4fingers, ulnar grasp) were selected for accomplishing this experiment, see Figure (1) (right). The subjects were instructed to keep the same orientation of their hands while performing these grasps. Each grasp type was repeated 30 times for a total of 150 trials per subject. The raw EMG data was filtered and normalized with the maximum voluntary contraction (MVC) and the Cyberglove was calibrated using the method described in [7]. The previous two data streams were synchronised via an Arduino board in order to analyze the EMG activity along with the hand motion.

\section{CLASSIFICATION RATE VS HAND PRE- SHAPE}

The recorded muscular activity was consistent across grasps for the same subject, and significantly different across different subjects. Therefore, for each subject, one specific Echo State Networks (ESN) classifier was trained [1]. The input to each ESN consists of a sequence of 15-dimensional EMG measurements recorded over a period of $150 \mathrm{~ms}$. We start classifying at $150 \mathrm{~ms}$ and perform classification at each time step with a sliding window of $100 \mathrm{~ms}$, see Figure (2).

For each subject, the data was divided into a training and a testing set constituting each $50 \%$ of the data, i.e 75 repetitions for training and 75 repetitions for testing. Figure (2) presents the classification results obtained for the tripod grasp strategy. We define the success rate as the percentage of EMG data correctly classified as a specific grasp type to the total number of reach-to-grasp motions corresponding to this specific grasp type. Across all subjects and grasp types, we obtained a success rate of $98 \%$ with a standard deviation of $2 \%$ at the end of the reaching and grasping motion.

Figure (2) also shows where the classification occurs with respect to the hand preshape. We use two typical measurement that characterize the temporal evolution of a grasp $[8,4]$, namely: 1) the area obtained by interconnecting the tips of the fingers active in the grasp [8] and 2) the hand aperture obtained as the distance between the thumb and the index [4] or the distance between the thumb and the pinky finger depending on which pair of fingers is used in the grasp. Notice that the preshape of the hand is obtained at the peak value of these criteria. If we consider the tripod grasp in Figure (2), a classification rate of $90 \%$ occurs at $0.2 \mathrm{sec}$ while the preshape of the hand occurs at $0.67 \mathrm{sec}$. A classification success rate of $90 \%$ is obtained for the precision disk grasp at $0.25 \mathrm{sec}$ while the hand preshape occurs at 0.8 sec. Similarly, for the ulnar pinch, a good classification of the data is obtained before the preshape of the hand. It is clear from these values that a good classification of the reach to grasp strategy occurs before the preshape of the hand. As for the thumb-2 fingers and thumb-4 fingers grasp types, the computed hand preshape criteria did not have consistently a clear peak and thus we could not relate the classification success rate to the hand preshape for these grasp types.

\section{CONCLUSION}

In this work, we reported on classification performance of an EMG based learning approach that decodes the grasp-
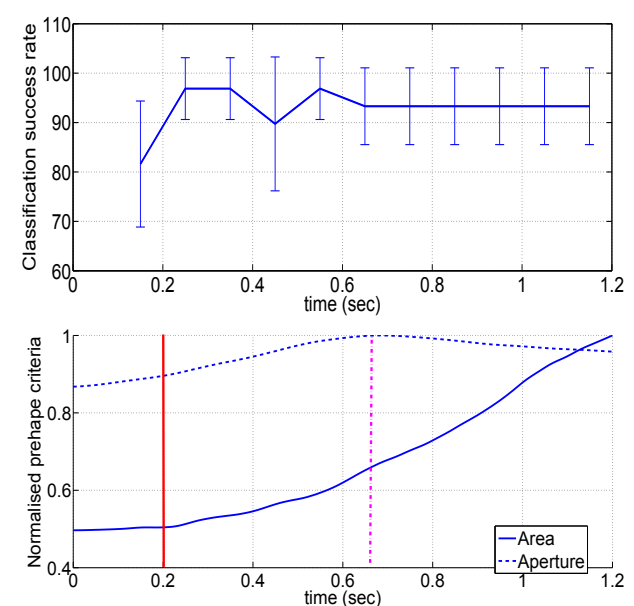

Figure 2: Tripod grasp: first plot shows the average and standard deviation of the classification rate across trials for subject 2. Second row plot shows the two computed preshape criteria, the dashed curve corresponds the hand aperture criterion while the solid curve corresponds to the area criterion. The vertical red line indicates a classification rate of $90 \%$ while the magenta dashed line corresponds to the hand preshape.

ing intention of the user at an early stage of the reaching to grasping motion. Our results show that a classification rate of $90 \%$ occurs clearly before the preshape of the hand for the precision disk, tripod and ulnar pinch. As for the thumb-2 fingers and thumb-4 fingers grasp types, the computed hand preshape criteria did not always show a peak and thus the preshape of the hand could not always be clearly detected.

\section{ACKNOWLEDGEMENTS}

The authors would like to thank Dr. Martina Coscia and Elvira Pirondini for their assistance in the set-up of the experiment. The research leading to these results has received funding from the Swiss National Science Foundation through the National Centre of Competence in Research (NCCR) in Robotics.

\section{REFERENCES}

[1] H. Jaeger, Echo state network, Scholarpedia, 2(9):2330, 2007.

[2] M. V. Liarokapis, P. K. Artemiadis, K. J. Kyriakopoulos, and E. S. Manolakos, A Learning Scheme for Reach to Grasp Movements: On EMG-Based Interfaces Using Task Specific Motion Decoding Models, in IEEE Journal of Biomedical and Health Informatics, vol. 17, no. 5, 2013.

[3] G. Ouyang, X. Zhu, Z. Ju, and H. Liu, Dynamical Characteristics of Surface EMG Signals of Hand Grasps via Recurrence Plot, in IEEE Journal of Biomedical and Helath Informatics, vol. 18, no. 1, 2014.

[4] Y. Paulignan, C. MacKenzie, R. Marteniuk, and M. Jeannerod, The coupling of arm and finger movements during prehension, Exp Brain Res, vol. 79, pp. 431-435, 1990.

[5] M. Santello, M. Flanders, and J. F. Soechting, Postural hand synergies for tool use, The Journal of Neuroscience, 1998.

[6] P. Shenoy, K. J. Miller, B. Crawford, and R. P. N. Rao, Online Electromyographic Control of a Robotic Prosthesis, in IEEE Transactions on Biomedical Engineering, vol. 55, no. 3, 2008.

[7] R.L. de Souza, R. L., S. El-Khoury, J. Santos-Victor, and A. Billard, Towards comprehensive capture of human grasping and manipulation skills, in proceedings of the Thirteenth International Symposium on the 3-D Analysis of Human Movement, 2014.

[8] T. Supuk, T. Kodek, T. Bajd, Estimation of hand preshaping during human grasping, Medical Engineering \& Physics, vol. 29, pp. 790-797, 2005 